\title{
Shared latent structures between imaging features and biomarkers in early stages of Alzheimer's disease: a predictive study
}

\author{
Adrià Casamitjana, Paula Petrone, José Luis Molinuevo, Juan Domingo Gispert and Verónica Vilaplana, \\ for the Alzheimer's Disease Neuroimaging Initiative
}

\begin{abstract}
Magnetic resonance imaging (MRI) provides high resolution brain morphological information and is used as a biomarker in neurodegenerative diseases. Population studies of brain morphology often seek to identify pathological structural changes related to different diagnostic categories (e.g: controls, mild cognitive impairment or dementia) which normally describe highly heterogeneous groups with a single categorical variable. Instead, multiple biomarkers are used as a proxy for pathology and are more powerful in capturing structural variability. Hence, using the joint modeling of brain morphology and biomarkers, we aim at describing structural changes related to any brain condition by means of few underlying processes. In this regard, we use a multivariate approach based on Projection to Latent Structures in its regression variant (PLSR) to study structural changes related to aging and AD pathology. MRI volumetric and cortical thickness measurements are used for brain morphology and cerebrospinal fluid (CSF) biomarkers (t-tau, $p$-tau and amyloidbeta) are used as a proxy for $A D$ pathology. By relating both sets of measurements, PLSR finds a low-dimensional latent space describing $A D$ pathological effects on brain structure. The proposed framework allows to separately model aging effects on brain morphology as a confounder variable orthogonal to the pathological effect. The predictive power of the associated latent spaces (i.e. the capacity of predicting biomarker values) is assessed in a crossvalidation framework.
\end{abstract}

Index Terms-Latent model, PLS, preclinical AD, CSF biomarkers, MRI

\section{INTRODUCTION}

Adrià Casamitjana and Verónica Vilaplana are with the Universitat Politècnica de Catalunya (UPC BarcelonaTech), Barcelona, Spain (email:adria.casamitjana, veronica.vilaplana@upc.edu)

Paula Petrone, José Luis Molinuevo and Juan Domingo Gispert, are with BarcelonaBeta Brain Research Centre (BBRC), Barcelona, Spain (e-mail: ppetrone,jlmolinuevo,jdgispert@barcelonabeta.org).

Data used in preparation of this article were obtained from the Alzheimer's Disease Neuroimaging Initiative (ADNI) database (adni.loni.usc.edu). As such, the investigators within the ADNI contributed to the design and implementation of ADNI and/or provided data but did not participate in analysis or writing of this report. A complete listing of ADNI investigators can be found at: http://adni.loni.usc.edu/wpcontent/ uploads/how to apply/ADNI Acknowledgement List.pdf.

This work has been partially supported by the project MALEGRA TEC2016-75976-R financed by the Spanish Ministerio de Economía y Competitividad and the European Regional Development Fund (ERDF). Adrià Casamitjana is supported by the Spanish Ministerio de Educación, Cultura y Deporte FPU Research Fellowship (FPU14/05988). Juan D. Gispert holds a 'Ramón y Cajal' fellowship (RYC-2013-13054)
$\mathbf{H}$ UMAN brains are constantly evolving throughout life, changing their neurobiological structure according to an uncountable number of factors ranging from genetics or hormonal to vascular factors [?]. Hence, we can define multiple interdependent processes occurring at the same time in each subject's brain. A normal aging process describe similar changes in groups of subjects with similar age and can be characterized by subject's real age. Often, in elderly subjects, other processes related to dementia occur in parallel and eventually result in cognitive or memory decline. The etiology of those processes is still unknown but a large literature of promising research is being published, specially for the Alzheimer's disease (AD), the most common type of dementia. The work in [?] goes through several examples of recent findings commenting the evidences found. In that sense, cerebrospinal fluid (CSF) biomarkers, as a proxy measure of pathophysiological (A $\beta$ and tau deposition) processes, show an acceptable sensitivity and specificity for AD diagnostic purposes and can be used as a measure of disease progression along the $\mathrm{AD}$ continuum [?]. This lead to the definition of the preclinical (PC) stage of $\mathrm{AD}$, where subjects present abnormal levels of $\mathrm{A} \beta$ in the brain without cognitive decline or clinical symptoms [?]. More recently, an alternative approach defining $\mathrm{AD}$ as a biological continuum rather than a syndrome has been proposed [?]. In this line, promising developments in PET imaging could provide us with more sensitive and specific pathological biomarkers [?]. On the other hand, high-field MR imaging provide detailed brain anatomical information as a measure of brain structure that can be used to study neurodegenerative processes associated with brain development and aging [?], cognitive decline [?], [?] and amyloid positivity [?]. The inter-relationship between AD pathological markers, neurodegeneration and cognition is highly studied in the literature [?], [?]. However, CSF and PET are costly and/or invasive biomarkers while structural MRI is a cheap and noninvasive biomarker of neurodegeneration. Hence, the overall goal of this work is to predict CSF biomarkers using MRI features and study their relationship. To this end, we consider two separate processes governing brain morphology along the $\mathrm{AD}$ continuum: aging and $\mathrm{AD}$, measured through age and CSF biomarkers, respectively. We acknowledge the presence of other processes (e.g: non-AD pathologies) specially in later stages of the disease, but these analyses are out of the scope 
of this work.

Structural brain changes are heterogeneous among subjects [?] and may not be uniform across the brain, specially if related to dementia. However, we hypothesize that these changes can be described by a small set of interdependent underlying processes that define common morphological patterns across all subjects. We build two different latent models to find specific effects due to brain aging and $\mathrm{AD}$ and couple them in a single brain structure model. Moreover, we constrain both models to be orthogonal to each other in order to disentangle aging from disease processes on brain morphology. We investigate and compare two different modeling approaches of $\mathrm{AD}$ effects: constant along the continuum or separate models for different stages. Projection to Latent Structures (also known as Partial Least Squares) is used in its regression form (PLSR) to jointly model the variation of MRI measurements as predictor variables and age and CSF biomarkers as response variables. Finally, we use the learned patterns for predicting age and AD pathological markers.

Related work: PLS modelling in any of its variants has been widely used in neuroimaging studies and a good review of several applications in the field can be found in [?]. As an example, PLS is used in [?] to jointly model the variation of genotype and phenotype in Alzheimer's disease, using single nucleotide polymorphisms (SNP) and 5 regional brain volumes as measurements. A white matter integrity analysis through diffusion tensor imaging (DTI) is analyzed in [?], where several diffusion parameters (axial diffusivity (AD), radial diffusivity (RD) and fractional anisotropy (FA)) are used to study differences in white matter between different $\mathrm{AD}$ stages. In [?], underlying brain morphometric patterns are used to predict subjects that convert from mild cognitive impaired (MCI) to AD-dementia with high predictive power. Similar to our work, the goal in [?] is to find latent spaces related to aging and disease processes using PLS to model the variability of brain shape features with age and clinical scores. Here, they jointly model aging and AD effects using age and the mini mental score examination (MMSE) cognitive test, respectively. In a cross-sectional analysis, they find overlap between aging and $\mathrm{AD}$ processes while using longitudinal data disentangles both effects. Other multivariate methods used for diagnosis classification using a wide range of imaging modalities are described in [?]. However, clinical scores or cognitive profiles (i.e MMSE) are better suited to assess dementia as a syndrome rather than to characterize the full spectrum of $\mathrm{AD}$. In that sense, CSF biomarkers are widely used as a proxy fo AD pathology [?]. Prediction of amyloid-positive subjects using MRI-based multivariate methods is an increasing area of research [?], [?] due to its potential applications in clinical screening. Studies relating CSF biomarkers and MRI measurements in the literature mostly use standard hypothesis testing and linear regression techniques. A comparison between CSF and MRI discriminative and predictive power can be found in [?] and [?], respectively. In the first, cross-sectional MRI and CSF measurements are used to predict different clinical groups along the disease continuum, while in the second they are used as baseline measurements to predict future clinical change measured through Clinical Dementia Rating- sum of boxes (CDR-SB) and MMSE. Both works show that MRI outperforms CSF but they show complementary information, providing a boost in performance when using both for classification and prediction. Finally, the work in [?] independently relates each CSF measure with brain ROIs using the General Linear Model and hypothesis testing showing moderate relationships between baseline CSF measurements and longitudinal atrophy rates in many brain structures.

\section{METHODS}

$\mathbf{I}$ $\mathrm{N}$ this work, we aim at predicting pathological markers (i.e: CSF biomarkers) using brain morphological (i.e. MRI) features. Our modeling assumptions that there coexist two main process in the brain (brain aging and AD processes) that can be described by means of few underlying structures related to brain morphometry. These latent patterns are further used to predict AD pathological markers. For this purpose, we use Partial Least Squares regression (PLSR) methodology [?].

\section{A. PLSR: Partial Least Squares Regression}

In general, Partial Least Squares (PLS) is a statistical model that analyzes the joint variability between two sets of measurements, ( $X$ and $Y$ ) on the same observations. Its main goal is to find latent structures related to both input measurements whose covariance is maximized. In the same line, the PLSR approach aims at predicting one set of measurements, $Y$ from the other $X$ by means of common latent structures that maximize the joint variability among them. Formally, PLSR finds two sets of weight vectors $(w, c)$ that jointly decompose the predictor matrix $X \in \mathcal{R}^{N \times K}$ and the response matrix $Y \in \mathcal{R}^{N \times M}$ into latent variables $(t, u)$ by maximizing the covariance of the latent spaces. Here, $N$ stands for the number of observations, while $K$ and $M$ define the number of predictors and response variables, respectively.

For a single latent dimension $(L=1)$ PLSR finds the underlying representations as linear combinations of input measurements $t=X w, u=Y c$ such that

$$
\begin{array}{ll}
\operatorname{maximize} & \operatorname{cov}(X w, Y c)=\mathcal{E}\left\{\left(t-t_{0}\right)\left(u-u_{0}\right)\right\} \\
& \text { s.t } \quad w^{T} w=1, c^{T} c=1
\end{array}
$$

Solving Eq. ?? using Lagrange multipliers, weight vectors have the following analytic expression:

$$
w=\frac{X^{T} u}{\sqrt{u^{T} X X^{T} u}}, \quad c=\frac{Y^{T} t}{\sqrt{t^{T} Y Y^{T} t}}
$$

For a latent space of dimension $L>1$, each subsequent latent factor is computed by iterating over the same optimization process (i.e. Eq.??,??) and concatenating the results $\left(W=\left[w_{0}, w_{1}, \ldots, w_{L-1}\right] \in \mathcal{R}^{K \times L}, C=\right.$ $\left[c_{0}, c_{1}, \ldots c_{L-1}\right] \in \mathcal{R}^{M \times L}, T=\left[t_{0}, t_{1}, \ldots, t_{L-1}\right] \in \mathcal{R}^{N \times L}, U=$ $\left.\left[u_{0}, u_{1}, \ldots, u_{L-1}\right] \in \mathcal{R}^{N \times L}\right)$. However, at each iteration, PLS uses deflated versions of input measurements ( $\mathrm{X}$ and $\mathrm{Y}$ ) as new predictors and response variables, forcing successive latent directions to be orthogonal to previous ones and hence, 
maximizing the input variance explained. The deflated version of $\mathrm{X}$ and $\mathrm{Y}$ at ith-iteration is:

$$
\begin{gathered}
X_{i+1}=X_{i}-t_{i} p_{i}^{T}=\left(I-\frac{t_{i} t_{i}^{T}}{t_{i}^{T} t_{i}}\right) X_{i}, \quad p_{i}=X_{i}^{T} t_{i} /\left(t_{i}^{T} t_{i}\right) \\
Y_{i+1}=Y_{i}-t_{i} q_{i}^{T}=\left(I-\frac{t_{i} t_{i}^{T}}{t_{i}^{T} t_{i}}\right) Y_{i}, \quad q_{i}=Y_{i}^{T} t_{i} /\left(t_{i}^{T} t_{i}\right)
\end{gathered}
$$

with $i=0, \ldots, L-2, X_{0}=X$ and $Y_{0}=Y$ Due to continuous deflation of input spaces, weight matrices $(W, C)$ do not directly relate input $(X, Y)$ and latent $(T, U)$ spaces. Accounting for that, rotation matrices, $R_{x}, R_{y}$, are defined:

$$
\begin{aligned}
& R_{x}=W \cdot\left(P^{T} \cdot W\right)^{-1}, \quad T=X \cdot R_{x}, \\
& R_{y}=C \cdot\left(Q^{T} \cdot C\right)^{-1}, \quad U=Y \cdot R_{y}
\end{aligned}
$$

where $P=\left[p_{0}, p_{1}, \ldots, p_{L-1}\right] \in \mathcal{R}^{K \times L}, Q=$ $\left[q_{0}, q_{1}, \ldots, q_{L-1}\right] \in \mathcal{R}^{M \mathrm{x} L}$ are the so-called loadings. Finally, PLSR modeling is used for predicting $Y$ from $X$ using the coefficient matrix $B$ :

$$
\begin{aligned}
& Y=T Q^{T}+E=X R_{x} Q^{T}+E \\
& Y=X B+E, \text { where } B=R_{x} Q^{T}
\end{aligned}
$$

where $E$ models the regression error.

\section{B. PLSR orthogonalization and coupling}

A common assumption in neuroimaging studies is that the object of study (e.g: brain morphology) is affected at the same time by the condition of interest (e.g: AD) and confounding variables (e.g: age, genetics). The standard solution is to regress-out the unwanted factors on the condition of interest. In a PLSR framework, it can be done estimating two separate models for confounders $\left(\mathrm{M}_{C}\right)$ and the variable of interest $\left(\mathrm{M}_{I}\right)$ and imposing orthogonality between models. Similarly to the work in [?], we introduce the orthogonality constraint in the optimization process forcing the associated latent subspace $\left(T_{C}, T_{I}\right)$ to be orthogonal. The $M_{C}$ model using $X, Y_{C}$ as predictor and response variables, respectively, is found by solving the regular expression in Eq. ?? for an $L_{C}$-dimensional subspace with weights $w_{C i}$ for $i=0: L_{C}-1$. To account for the variance explained by confounding variables, we subtract the measurement variance explained by $M_{C}$ in the predictor variable of $\mathrm{M}_{I}$, resulting in a deflated version of the input measurement $\bar{X}=X-T_{C} P_{C}^{\top}$. Hence, the optimization process for $\mathrm{M}_{I}$ model using $Y_{i}$ as response variables is as follows:

$$
\operatorname{maximize} \operatorname{cov}\left(\bar{X} \cdot w, Y_{I} \cdot c\right)=\mathcal{E}\left\{\left(t-t_{0}\right)^{\top} \cdot\left(u-u_{0}\right)\right\}
$$

$$
\text { s.t } \quad w^{T} w=1, c^{T} c=1, w^{T} \cdot r_{C i}=0 \quad \forall=0, \ldots, L_{C}-1
$$

where $r_{C i}$ are the columns of the PLSR rotation matrix $R_{C}$ of model $M_{C}$. For an $L_{I}$-dimensional subspace the resulting weights can be expressed as:

$$
\begin{aligned}
& w_{j}=\bar{X}_{j}^{\top} \cdot u_{j}-\sum_{i=0}^{L_{C}-1} \frac{r_{C i}^{\top} \cdot \bar{X}_{j}^{\top} \cdot u_{j}}{r_{C i}^{\top} \cdot r_{C i}} r_{C i} \Rightarrow w_{j}=\frac{w_{j}}{\left\|w_{j}\right\|} \\
& c_{j}=Y_{I j}^{\top} \cdot t_{j} \Rightarrow c_{j}=\frac{c_{j}}{\left\|c_{j}\right\|} \quad j=0: L_{I}-1
\end{aligned}
$$

where $\overline{X_{j}}$ and $Y_{I j}$ are the deflated version of $\overline{X_{0}}$ and $Y_{I 0}$ at $j$ th iteration. Finally, the full PLSR model $\left(\mathrm{M}^{\text {full }}, L^{\text {full }}\right.$ dimensional) is built by concatenating latent scores and rotation matrices from both models: $T=\operatorname{concat}\left(t_{l}\right)$ and $R=$ $\operatorname{concat}\left(R_{l}\right)$ where $l=0, \ldots, L_{C}-1, \ldots, L_{C}+L_{I}-1$

\section{Model definition: $\mathrm{M}^{\text {aging }}, \mathrm{M}^{\mathrm{AD}}$}

We define two separate models for brain aging and $\mathrm{AD}$ effects $\left(\mathrm{M}_{C}=\mathrm{M}^{\text {aging }}, \mathrm{M}_{I}=\mathrm{M}^{\mathrm{AD}}\right)$. We first estimate $\mathrm{M}^{\text {aging }}$ model using normal aging subjects and brain volumetric features as predictor and age as response variable. Thereafter, we use the methodology state in section ?? to fit the $\mathrm{M}^{\mathrm{AD}}$ model using subjects in the AD pathophysiological path and a deflated version of brain volumetric features as predictor and CSF biomarkers $(\mathrm{A} \beta$, $\mathrm{p}$-tau, $\mathrm{t}$-tau $)$ as response variables. The final latent space $\left(M^{\text {full }}\right)$ is built by concatenating the latent model associated with brain aging and the latent model associated with AD markers.

\section{Statistical inference}

The outcome measures of interest using the aforementioned PLSR model are: (i) the effect strength $\rho_{l(s)}$, defined as the Pearson correlation of the $l$ th latent factor and a certain variable of interest $s$ (e.g. CSF biomarkers, age) and (ii) the effect type, $\nu_{l}$ defined as the projection from input X-space to its related latent space.

$$
\rho_{l(s)}=\frac{\operatorname{cov}\left(t_{l}, y_{s}\right)}{\sigma_{t_{l}} \cdot \sigma_{y_{s}}} \quad \nu_{l}=r_{l} \quad l=0, \ldots, L^{\text {full }}-1
$$

For statistical inference over the outcome measures of the PLSR model, non-parametric permutation testing is used [?]. The goal is to find latent patterns that are relevant for brain condition variables $(s)$. The null hypothesis states that there is no correlation between $l$ th latent factor of the model and response variable $s$ and is estimated by breaking the initial relationship between brain morphology (X) and CSF biomarkers (Y). It can be done by permuting subject indices on $\mathrm{Y}$ measurements and using this new dataset $\left(\mathrm{X}, \mathrm{Y}_{\text {perm }}\right)$ to fit a PLSR model. The resulting latent space is used to compute the effect strength for each AD biomarker and age. This procedure is repeated $\mathrm{N}_{\text {perm }}$ times to estimate the sampling distribution for each variable $(s)$ under the null hypothesis.

\section{EXPERIMENTAL ANALYSIS}

\section{A. Data}

In our experiments we use the publicly available dataset from the Alzheimer's Disease Neuroimaging Initiative ${ }^{1}$. We

\footnotetext{
${ }^{1}$ http://adni.loni.usc.edu
} 
build a sample of $N=802$ subjects split into diagnostic categories following recently published guidelines [?]: $N_{H C}=$ 189 healthy controls (HC), $N_{P C}=136$ preclinical (PC) subjects, $N_{M C I}=330$ subjects labeled as mild cognitive impairment (MCI) and $N_{A D-\text { dementia }}=147$ subjects diagnosed with dementia due to $\mathrm{AD}$. MCI and $\mathrm{AD}$-dementia subjects are diagnosed following the standard criteria used in ADNI and reported in [?] while for PC subjects we select asymptomatic subjects with positive amyloid-beta using the extensively used threshold at $\mathrm{A} \beta<192 \mathrm{pg} / \mathrm{mL}$ [?]. All subjects have a T1weighted MRI available, preprocessed using FreeSurfer ${ }^{2}$ and segmented according to [?] and [?] into regions of interest (ROIs). Grey matter volume divided by the total intracranial volume and cortical thickness averages are computed for each ROI and used as predictor variables (X). Age and CSF biomarkers $(\mathrm{A} \beta$, $\mathrm{p}$-tau, $\mathbf{t}$-tau) are used as response variables (Y). Together with age and CSF biomarkers, a normalized CSF index as diagnostic metric for disease progression (AD-CSF, [?]) is also used for the analysis of AD pathological effects on the latent model. The AD-CSF index is a combination of CSF biomarkers and has two different forms: (i) AD-CSF1 that involves $\mathrm{p}$-tau protein and $\mathrm{A} \beta$ and (ii) $\mathrm{AD}-\mathrm{CSF} 2$ that involves $\mathrm{t}$-tau and $\mathrm{A} \beta$.

We use the linear regression model in Eq. ?? to define the dimension of each latent space, $L$, by evaluating the total mean absolute error (MAE) of the predicted response variables in a 5-fold cross-validation framework [?]. To model brain aging, we use only $\mathrm{HC}$ subjects and set $L^{\text {aging }}=2$ while for modeling brain $\mathrm{AD}$ we investigate two a priori hypothesis: (i) latent patterns (effect-type) governing $\mathrm{AD}$ pathology are linear across all continuum with possibly different effect strengths at different stages and (ii) latent patterns governing $\mathrm{AD}$ pathology are stage-wise linear across the continuum. For the first case, we model brain $\mathrm{AD}$ with $L^{\mathrm{AD}}=4$ using PC, $\mathrm{MCI}$ and $\mathrm{AD}$-dementia subjects together in one single model, while for the second case we model brain AD separately at each stage resulting in 4 different models for NC, PC, MCI and $\mathrm{AD}$-dementia subjects $\left(L^{\mathrm{AD}}=4\right.$-dimensional model for each stage). The overall latent space in both cases is built by concatenating latent factors from the brain aging model $\left(t_{0}, t_{1}\right)$ and from $\mathrm{AD}$ model $\left(t_{2}, t_{3}, t_{4}, t_{5}\right)$. To refer to subspaces found using volumetric or cortical thickness data we use $t_{i}^{V}$ and $t_{i}^{C T}$, respectively. We investigate the effect strength of each latent dimension with age and $\mathrm{AD}$ pathological markers and assess their relevance using permutation testing with $N_{\text {perm }}=1000$ permutations and an uncorrected threshold of $\mathrm{p}<0.05$. The associated brain morphometric patterns for aging and $\mathrm{AD}$ processes are shown and compared with the literature. Finally, we show the predictive power of each model using a 5 -fold cross-validation framework to report the mean absolute error (MAE) of each variable of interest.

\section{B. Single model for AD pathology}

Using a single model for all disease stages, we assume that the effect type of dementia is preserved throughout the $\mathrm{AD}$ continuum even though the effect strength might change

\footnotetext{
${ }^{2}$ https://surfer.nmr.mgh.harvard.edu/
}

at each stage. The effect strength of brain condition markers on each latent factor $\left(\rho_{l(s)}\right)$ is shown in figure ?? and listed in Tables ??,?? (Appendix ??) with the associated p-values for models using volumetric and cortical thickness features, respectively.

To better understand the model of each measurement (volumetric and cortical thickness), we first analyze the effect strength related to the variables of interest at each latent dimension $\rho_{l(s)}$. In figure ?? we show the absolute value of the correlation between each latent variable $t_{l}$ and the respective indicators $s=$ age, CSF biomarkers and the two AD-CSF indices evaluated separately on subjects at specific AD stages (HC, PC, MCI, AD-dementia).

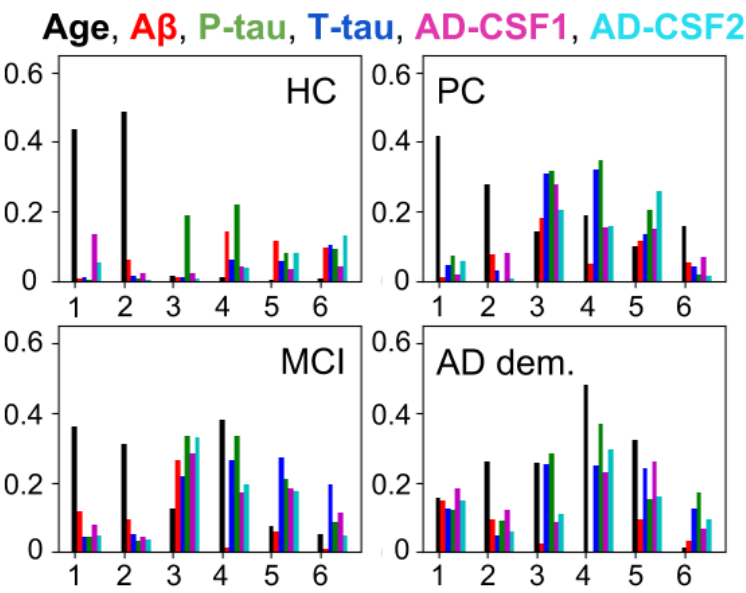

(a) Volume

Age, A $\beta$, P-tau, T-tau, AD-CSF1, AD-CSF2
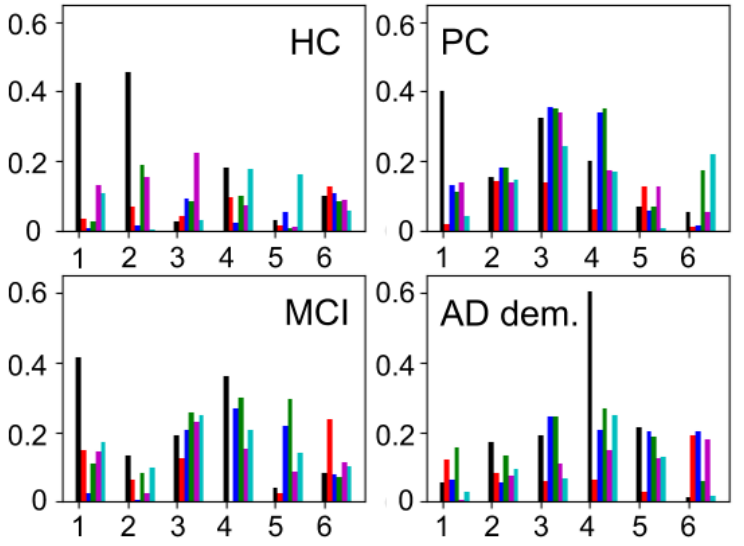

(b) Cortical Thickness

Fig. 1: Absolute value of the effect strength (single model) on several indicators evaluated at different stages: age (black), amyloid-beta (red), p-tau (green), t-tau (blue), AD-CSF-1 (magenta), AD-CSF-2 (cyan). Latent variables 0 and 1 correspond to brain aging model and latent variables $2,3,4$ and 5 correspond to brain $\mathrm{AD}$ and are shown in the x-axis.

Each effect type can be seen as a morphological pattern responding to certain conditions. According to the results in Tables ??,?? (Appendix ??) we can distinguish three different types of relevant $(\mathrm{p}<0.05)$ patterns along the $\mathrm{AD}$ continuum: (i) age-related patterns associated with high correlation with 
age, (ii) AD-related patterns that present high correlation with $\mathrm{AD}$ markers and (iii) mixed age and AD-related patterns that show high correlation values with age and several indicators of $\mathrm{AD}$ progression. The first one is found relevant on normal aging subjects, while the other two coexists along the $\mathrm{AD}$ continuum, meaning that aging and $\mathrm{AD}$ processes might partially overlap across the brain. We show an example of each type of pattern for volumetric features in figure ?? and for cortical thickness features in figure ??, in line with the corresponding effect strengths at each stage.

In figure ?? we show an age related pattern with high effect strength at pre-dementia stages (HC, PC and MCI) but low effect strength at AD-dementia stage. This pattern is estimated using only $\mathrm{HC}$ subjects and $\mathrm{M}^{\text {aging }}$ model and shows whole brain atrophy except for the choroid plexus regions. An AD-related pattern $\left(t_{2}\right)$ is depicted in figure ?? showing high correlation with AD pathological markers both on subjects along the whole AD continuum and independently at all stages. It shows a highly symmetric pattern were positive and negative values indicate neurodegeneration and increased ROI volume, respectively, due to its positive correlation with CSF-A $\beta$ and negative correlation with CSF-ptau and CSF-ttau values. Cortical regions (parahippocamapus, middle temporal, transverse temporal, inferior temporal) and subcortical regions (hippocampus, amygdala and fusiform) of the temporal lobe as well as regions such as the choroid plexus and, specially, the entorhinal cortex are the most affected regions positively correlated with $t_{2}$, indicating neurodegeneration over the course of $\mathrm{AD}$. Other regions such as the precentral or the pars orbitalis are negatively correlated with $t_{2}$. Finally, a mixed age and ADrelated pattern $\left(t_{3}\right)$ is shown in figure ??, which is negatively correlated with age and positively correlated with CSF-ptau, CSF-ttau and their associated indices along the AD continuum and independently at each stage while it remains independent of CSF-A $\beta$ values. Among the regions driving this pattern, we found choroid plexus, precuneus (negatively correlated with $t_{3}$ ), temporal pole and parahippocampus (positively correlated with $t_{3}$ ) the most relevant ones, indicating that they are involved in both brain aging and brain AD processes.

Similarly, patterns associated with cortical thickness are shown in figure ?? and are also split into age-related, ADrelated and mixed age and AD-related effects. Firstly, figure ?? presents an age-related pattern involving overall cortical reduction with age in the normal aging model $\mathrm{M}^{\text {aging }}$ except for the cingulate regions that show increased thickness once corrected by the total intracranial volume. Secondly, an ADrelated pattern is depicted in figure ??, involving cortical shrinkage of temporal regions (superior, middle, inferior) and the entorhinal cortex and increased thickness at regions such as precentral or transverse temporal. Finally, an age-AD mixed related pattern with opposite relationship between age (-) and CSF p-tau/t-tau (+) is shown in figure ??. It is associated with cortical shrinkage of parietal lobe regions and precuneus and increased thickness on parahippocampus, precentral, pars orbitalis, insula and superior temporal on the left hemisphere. Overall, similar findings are found for cortical thickness and volumetric data.

Finally, we evaluate the predictive power of the latent factors found for both age and CSF biomarkers at different disease stages. For better comparison, all indicators are globally standardized using $\mathrm{z}$-score on all subjects (i.e. mean centered and normalized by variance). Table ?? shows the mean absolute error (MAE) for each class using cortical thickness and volumetric features and can be interpreted as the fraction of error per standard deviation. These results can be compared with standard linear regression method found in figures ??,?? (Appendix ??).

\begin{tabular}{c|c|cccc}
\hline Features & Diagnosis & Age & CSF A $\beta$ & CSF p-tau & CSF t-tau \\
\hline Volume & HC & 0.49 & 1.91 & 0.74 & 0.71 \\
& PC & 0.68 & 0.46 & 0.72 & 0.63 \\
& MCI & 0.67 & 0.4 & 0.7 & 0.72 \\
& AD & 0.85 & 0.37 & 0.88 & 0.94 \\
\hline Cortical Th. & HC & 0.55 & 1.94 & 0.7 & 0.63 \\
& PC & 0.76 & 0.48 & 0.68 & 0.66 \\
& MCI & 0.77 & 0.42 & 0.69 & 0.72 \\
& AD & 0.91 & 0.38 & 0.87 & 0.88 \\
\hline \hline
\end{tabular}

TABLE I: Predictive error of response variables for each diagnosis label and feature type using a single model for all AD continuum.

Age is best predicted using $\mathrm{HC}$ subject and it increases with later stages at the $\mathrm{AD}$ continuum, showing that features affected by age are also affected by the disease. CSF A $\beta$ biomarker presents lower predictive error at $\mathrm{AD}$ continuum stages and high predictive error at HC stage probably due to the standardization process, where it presents low variance at $\mathrm{AD}$ continuum stages and higher variance at $\mathrm{HC}$ stage. CSF p-tau/t-tau seem not very predictable using brain morphometry measurements. Compared to standard linear regression, we found no clear gain of our methodology for the regression task. Hence, even though encountered patterns are highly related with certain conditions, they are not predictive of AD markers.

\section{Multiple models for AD pathology}

Under the assumption that underlying processes governing brain structure along the $\mathrm{AD}$ continuum might differ between stages, we fit a brain AD model independently for each stage including (i) HC, (ii) PC, (iii) MCI and (iv) AD-dementia. Similarly to section ??, we perform the analysis for volumetric and cortical thickness measurements and show the effect strength of each brain latent model with respect to several variables of interest at their corresponding stage (figure ??). A complete list of all effects strengths with their associated p-values is listed in Tables ??,?? (Appendix ??)

Underlying patterns from the brain aging model remain the same while latent factors related to brain $\mathrm{AD}$ model increase their effect strength and statistical relevance at each stage. More specific patterns of brain $\mathrm{AD}$ are found for each $\mathrm{AD}$ stage. Here, we focus on AD-related latent factors found in previous section (Sec. ??) to discover differences between brain morphological patterns related to $\mathrm{AD}$ at specific stages. Hence, in figure ?? and figure ?? we show the effect-type of the third latent dimension using volumetric $\left(t_{2}^{V}\right)$ and cortical thickness features $\left(t_{2}^{C T}\right)$, respectively. Both figures show the highest correlation with $\mathrm{AD}$ pathological markers. 

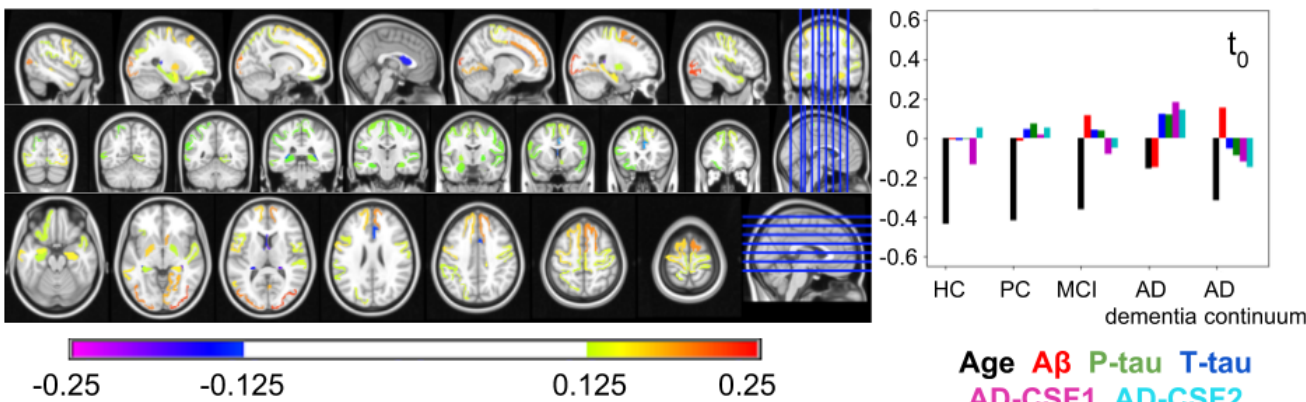

(a) Age-related pattern corresponding to the first latent dimension of the model $t_{0}^{V}$
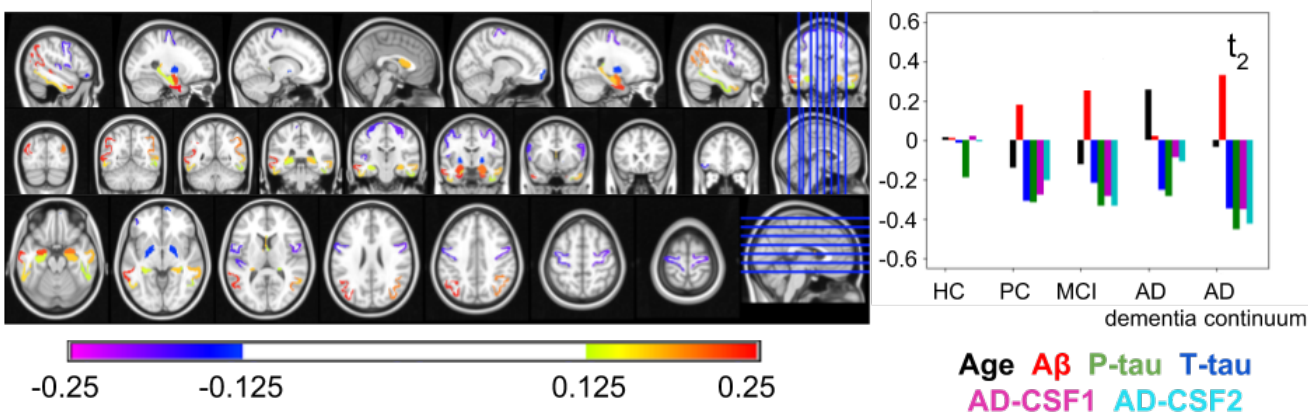

(b) AD-related pattern corresponding to the third latent dimension of the model $t_{2}^{V}$
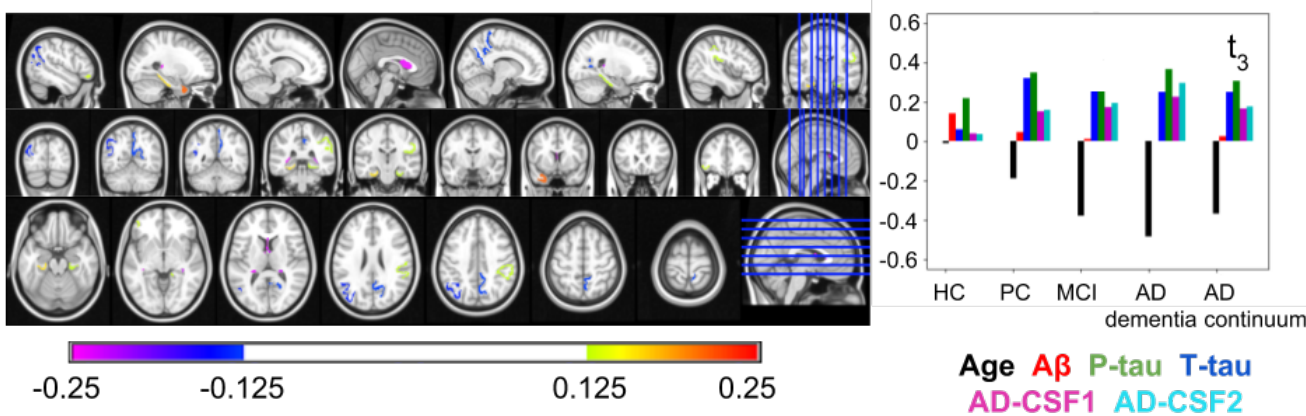

(c) Mixed age and AD-related pattern corresponding to the fourth latent dimension of the model $t_{3}^{V}$

Fig. 2: Examples of relevant latent brain morphological patterns (effect-type) using volumetric features. Values at each ROI represent their influence to the latent space. The corresponding effect strength of each condition at different stages of the disease is shown on the right.

Here, we concretely study the third dimension of the model $\left(t_{2}\right)$, an AD-related process that presents the highest correlation with $\mathrm{AD}$ pathological markers with both cortical thickness and volumetric features. In figure ?? we present the underlying pattern found at each stage of the disease using volumetric features. Compared to effect type found in previous analysis (figure ??), the effect type on MCI and AD-dementia stages appears to be very similar with the addition of the pallidum showing compensatory effect in the later. On the other hand, in the PC stage slightly differs from the global $\mathrm{AD}$ pattern yielding an specific preclinical signature. The neurodegeneration pattern involve choroid plexus and pallidum while pericalcarine show increased mean ROI volume. Again, similar results are found addressing volumetric and cortical thickness features.

For predictive analysis, all indicators are globally standardized using $\mathrm{z}$-score on all subjects (i.e. mean centered and normalized by variance). In Table ?? we summarize the mean absolute error (MAE) per class that can be interpreted as the fraction of error per standard deviation. Even though stage-wise modeling provides a higher effect strength and more relevant brain $\mathrm{AD}$ patterns, the predictive power of CSF biomarkers is slightly worse for late stages of the AD continuum, probably indicating a lower generalization power of the brain features found for the same number of latent dimensions. Nonetheless, PLSR has higher predictive power than the standard linear regression using raw morphometric features (Table ?? from Appendix ??).

\section{DISCUSSION}

In this work we aim at predicting $\mathrm{AD}$ pathological markers from brain morphology in elderly adults. To this end, we disentangle aging and pathological processes by orthogonal projections in a lower-dimensional subspace describing brain 


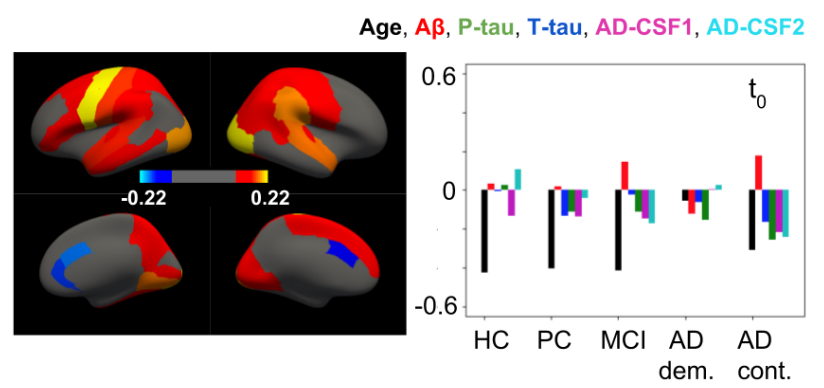

(a) Age-related pattern corresponding to the first latent dimension of the model $t_{0}^{C T}$

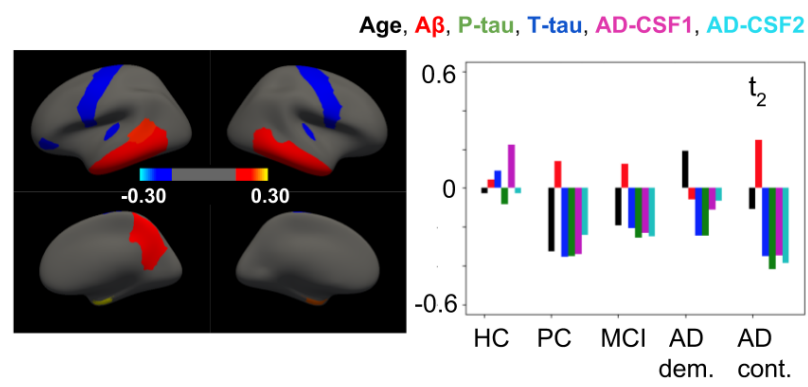

(b) AD-related pattern corresponding to the third latent dimension of the model $t_{2}^{C T}$

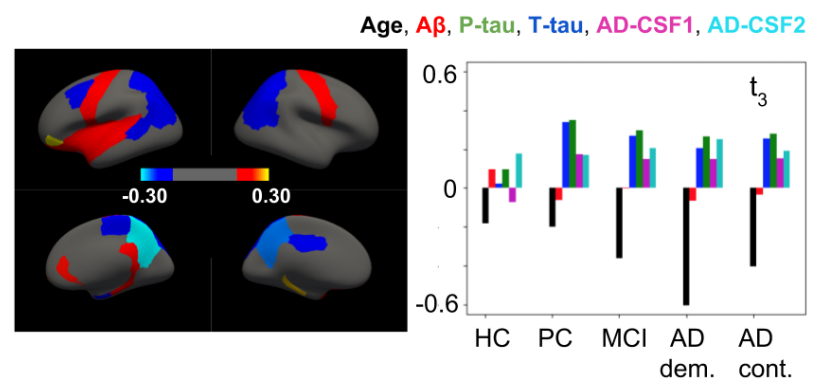

(c) Mixed age and AD-related pattern corresponding to the third latent dimension of the model $t_{3}^{C T}$

Fig. 3: Examples of relevant latent brain morphology patterns (effect-type) using cortical thickness features.Values at each ROI represent their influence to the latent space. The corresponding effect strength of each condition at different stages of the disease is shown on the right.

morphology along the disease continuum. We provide two different approaches to model the AD continuum: linear and stage-wise linear models, assuming in the later that brain anatomy might differ between AD stages. Statistical significance and predictability capacity of each model is assessed using either grey-matter volume or cortical thickness measurements as brain morphological features. Joint modeling of both types of features do not reveal further insights since the overall effect and predictive power remain similar indicating that they might not provide sufficient complementary information.

Both linear and stage-wise linear models find relevant patterns related to age, $\mathrm{AD}$ and a mixture of both, meaning that aging and disease processes might overlap, specially significant for later stages of the disease (MCI, AD-dementia). Using a single model for the whole AD continuum find common structures that are affected over the course of the disease, delineating the $\mathrm{AD}$ signature supported in the literature [?].
Age, A $\beta, P$-tau, T-tau, AD-CSF1, AD-CSF2
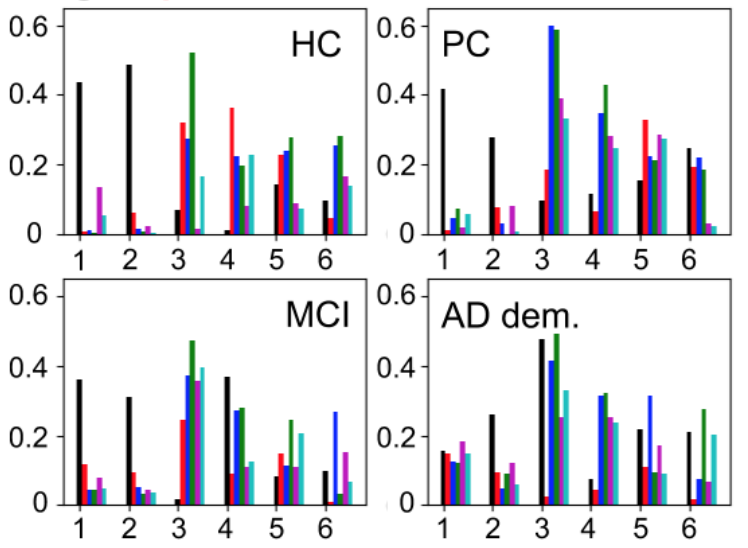

(a) Volume

Age, A, $\mathrm{P}$-tau, T-tau, AD-CSF1, AD-CSF2
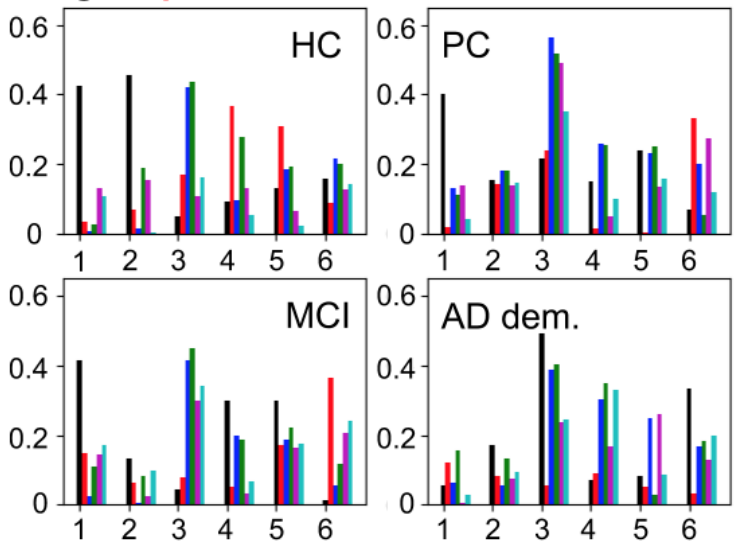

(b) Cortical Thickness

Fig. 4: Absolute value of the effect strength (multiple models) on several indicators evaluated at the respective disease stages: age (black), amyloid-beta (red), p-tau (green), t-tau (blue), AD-CSF-1 (magenta), AD-CSF-2 (cyan). Latent variables 0 and 1 correspond to brain aging model and latent variables 2 , 3,4 and 5 correspond to stagewise brain $\mathrm{AD}$ and are shown in the $\mathrm{x}$-axis.

On the other hand, fitting a model to each disease stage allow to find temporally specific patterns and increase the effect strength of $\mathrm{AD}$ pathological markers at each stage. While a single model find relevant patterns involving typical ADrelated regions in the temporal lobe, using separate stagewise linear models allows to uncover significant structures at specific stages. Using multiple models we could fit a separate model for each stage along the AD continuum increasing the correlation between descriptive brain morphological patterns and $\mathrm{AD}$ pathological markers. In detail, we found that brain structure at $\mathrm{MCI}$ and $\mathrm{AD}$-dementia stages relieve morphological patterns overlapping with the previously found AD signature. However, at preclinical (PC) stage the join variability of CSF biomarkers and brain structure follow a different and more specific pattern and thus, motivating the separate study of a preclinical $\mathrm{AD}$ signature, as several recent studies point 
Age A $\beta$-tau T-tau AD-CSF1 AD-CSF2

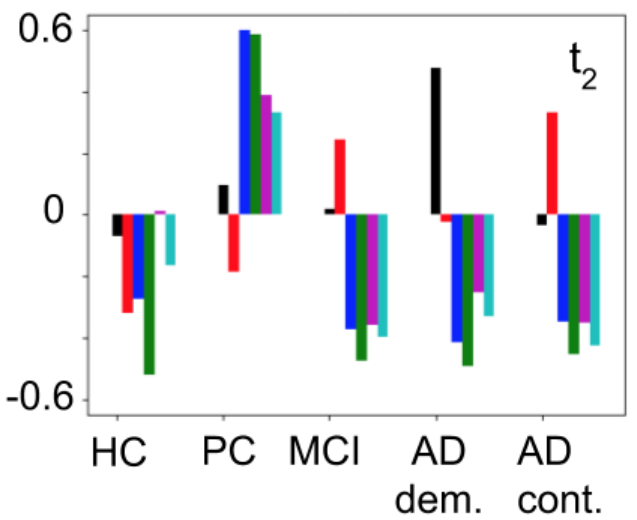

(a) Effect strength

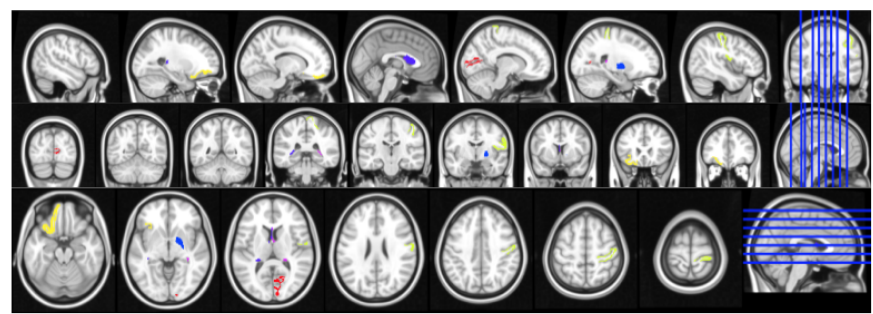

(b) PC stage

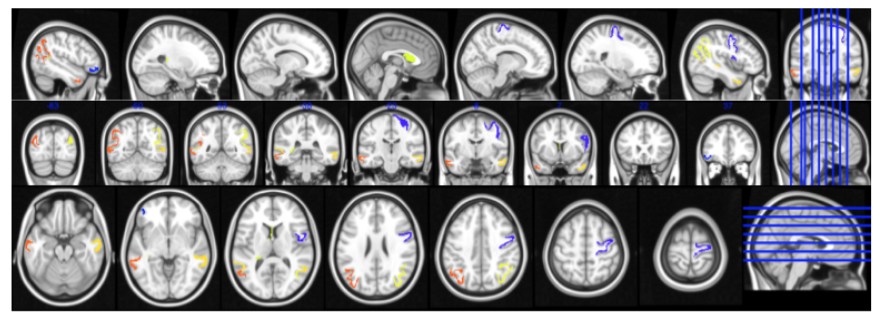

(c) MCI stage

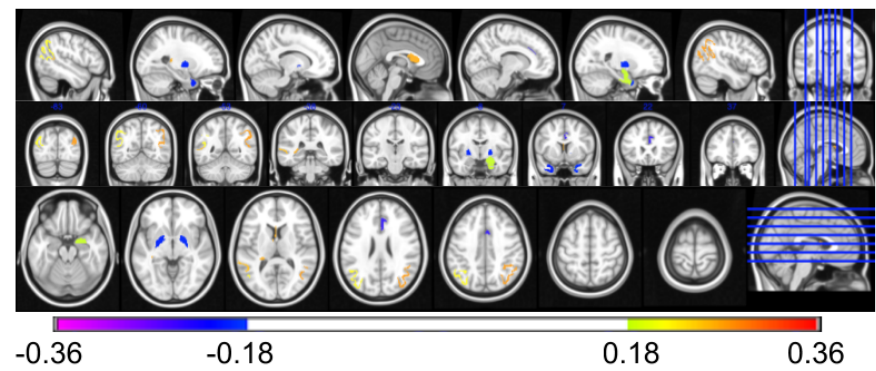

(d) AD stage

Fig. 5: AD-related effect-type of the brain latent model using cortical thickness features. It corresponds to the third latent dimension of the model $t_{2}^{V}$ at each stage of the AD continuum. The effect strength at each disease stage is shown on the top.

out [?], [?]. Interestingly, the pallidum effect over the course of $\mathrm{AD}$ is only detected using stage-wise modelling since it shows early degeneration but then it remains unaffected by the course of the disease. Some other structures, such as pericalcarine at PC stage or pallidum at AD-dementia stage, showed increased volume/thickness once corrected by age and the intracranial

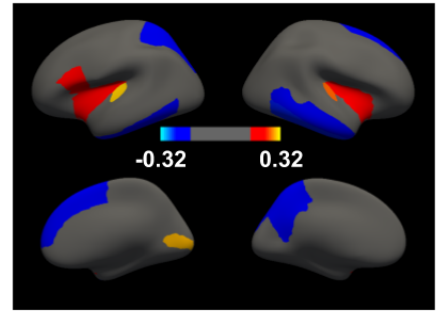

(a) PC stage

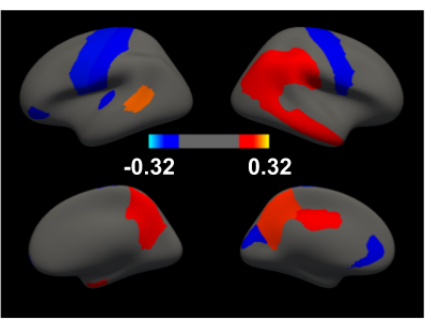

(c) AD stage

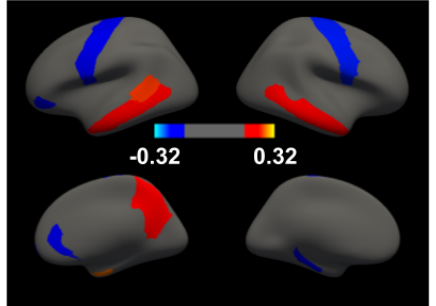

(b) MCI stage

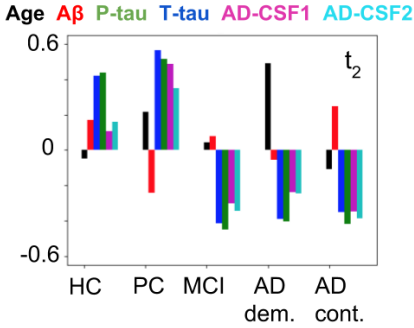

(d) Correlation
Fig. 6: AD-related effect-type of the brain latent model using cortical thickness features. It corresponds to the third latent dimension of the model $t_{2}^{C T}$ at each stage of the $\mathrm{AD}$ continuum. The effect strength at each disease stage is shown on the bottom-right.

\begin{tabular}{c|c|cccc}
\hline Features & Diagnosis & Age & CSF-A $\beta$ & CSF-ptau & CSF-ttau \\
\hline Volume & HC & 0.49 & 0.51 & 0.38 & 0.34 \\
& PC & 0.68 & 0.5 & 0.75 & 0.58 \\
& MCI & 0.67 & 0.41 & 0.72 & 0.75 \\
& AD & 0.85 & 0.33 & 1.07 & 1.0 \\
\hline Cortical Th. & HC & 0.55 & 0.54 & 0.36 & 0.36 \\
& PC & 0.76 & 0.48 & 0.73 & 0.61 \\
& MCI & 0.77 & 0.43 & 0.7 & 0.77 \\
& AD & 0.91 & 0.34 & 0.98 & 1.05 \\
\hline \hline
\end{tabular}

TABLE II: Predictive error of response variables for each diagnosis label and feature type using a separate model for each AD stage.

volume, which might be interpreted as compensatory effects due to increasing brain activity at those regions [?], [?].

Moreover, we provide a confounder model orthogonal to the AD continuum model that attempt to describe the brain aging process. It is estimated using only healthy control subjects and presents consistent results with the literature [?], [?], [?] for both volumetric and cortical thickness features involving global atrophy and cortical thinning, except for the choroid plexus region. This pattern appear to be highly correlated predementia stages (HC, PC, MCI) but has low effect strength at the dementia stage, providing more evidence of a higher overlap between aging and disease processes at later stages.

Finally, we assess the predictive power of both models and compare to standard linear regression model. Using stagewise modeling increases the overfitting at each stage, but the effect is mitigated using PLSR compared to linear regression. However, using a single model for the whole AD continuum provides similar results for both methods. Overall, the predictive performance is moderate to low and depends on the 
disease stage, being worse at later stages (AD-dementia).

Our results highlight the potential of using multivariate models to better detect and disentangle aging and $\mathrm{AD}$ pathological effects on brain structure instead of using univariate regression models and hypothesis testing studying each brain region independently [?]. Multivariate models are able to capture relevant and spatially distributed patterns of structural changes in the brain. In this work, we identify patterns of common variability between AD pathology, defined using CSF biomarkers, and brain structure, defined using either volumetric or thickness features. Throughout the literature, many multivariate approaches define AD pathology with binary or categorical variables (e.g: clinical diagnosis [?], [?] or amyloid status [?], [?]) and use machine learning as a multivariate model. However, the quantization of $\mathrm{AD}$ pathology in few categories reduces the modeling capacity of its variability and heterogeneity. To account for that, other works use single continuous clinical scores [?], [?], which is suited for modeling the later stages of the disease. Instead, CSF biomarkers, as used in our approach, are shown to better describe the full spectrum of $\mathrm{AD}$ [?]. Another recent multivariate approach to detect brain changes uses an event-based model [?] that clusters subjects depending on spatially distributed and temporally aligned brain changes, being well-suited for e.g. patient staging. However, they make a set of assumptions such that biomarkers vary independently and do not make use of underlying $\mathrm{AD}$ pathological markers. Instead of finding a sequence of events, in this work we could identify few underlying patterns of brain variability that are maximally related to $\mathrm{AD}$ pathology finding three different types of patterns: age-related, AD-related and mixed age and $\mathrm{AD}$ related.

\section{CONCLUSIONS}

We present a multivariate framework that describes structural changes related two different processes that may occur simultaneously in elderly subjects: brain aging and brain AD. Age and CSF biomarkers describe each process, respectively, and brain morphological features are used to assess brain structural information. PLSR is used to find latent spaces related to each brain condition and we incorporate a orthogonality constraint between both subspaces. Results show that along the $\mathrm{AD}$ continuum brain patterns related to age, $\mathrm{AD}$ and a mixture of both coexist which might indicate that brain aging and $\mathrm{AD}$ processes partially overlap. As largely investigated in the literature, we found that temporal lobe changes occur earlier in the disease continuum and are followed by later changes in parietal regions. Moreover, some regions of the brain present compensation effects once brain aging is considered. AD pathology expresses similarly in volumetric and cortical thickness features. One limitation of the model is that localized interpretations of condition effects (e.g. aging, dementia) in independent ROIs is not possible and the only conclusion that can be drawn is related to the overall morphometric patterns found. Future work may involve considering AD comorbidities and incorporating other imaging modalities in the study.

\section{APPENDIX I \\ LINEAR REGRESSION FOR PREDICTION}

We use linear regression with raw brain morphometric features as a baseline method for predictive analysis. Linear regression uses raw features to predict $\mathrm{AD}$ pathological markers and age. Tables ?? and ?? summarize the results using a single model for the whole AD continuum and a model for each $\mathrm{AD}$ stage, respectively.

\begin{tabular}{c|c|cccc}
\hline Features & Diagnosis & Age & CSF-A $\beta$ & CSF-ptau & CSF-ttau \\
\hline Volume & HC & 0.5 & 1.91 & 0.8 & 0.72 \\
& PC & 0.69 & 0.51 & 0.74 & 0.71 \\
& MCI & 0.68 & 0.43 & 0.74 & 0.73 \\
& AD & 0.85 & 0.41 & 0.93 & 0.89 \\
\hline Cortical Th. & HC & 0.53 & 1.89 & 0.77 & 0.76 \\
& PC & 0.72 & 0.51 & 0.73 & 0.78 \\
& MCI & 0.74 & 0.43 & 0.71 & 0.78 \\
& AD & 0.88 & 0.4 & 0.96 & 0.99 \\
\hline \hline
\end{tabular}

TABLE III: Predictive error of response variables for each diagnosis label and feature type using a single linear regression model for all AD continuum.

\begin{tabular}{c|c|cccc}
\hline Features & Diagnosis & Age & CSF-A $\beta$ & CSF-ptau & CSF-ttau \\
\hline Volume & HC & 0.5 & 0.67 & 0.58 & 0.49 \\
& PC & 0.69 & 0.88 & 1.4 & 0.86 \\
& MCI & 0.68 & 0.47 & 0.8 & 0.8 \\
& AD & 0.85 & 0.63 & 1.82 & 1.7 \\
\hline Cortical Th. & HC & 0.53 & 0.67 & 0.46 & 0.47 \\
& PC & 0.72 & 0.78 & 1.41 & 0.96 \\
& MCI & 0.74 & 0.46 & 0.76 & 0.83 \\
& AD & 0.88 & 0.58 & 1.4 & 1.35 \\
\hline \hline
\end{tabular}

TABLE IV: Predictive error of response variables for each diagnosis label and feature type using linear regression fitting a specific model for each $\mathrm{AD}$ stage.

\section{APPENDIX II \\ STATISTICAL INFERENCE TABLES}

We provide numbers of effect-strength and associated $\mathrm{p}$ value of $\mathrm{AD}$ pathological markers and age on the brain morphological latent model at different $\mathrm{AD}$ stages in Tables ??,??,??,??. 


\begin{tabular}{|c|c|c|c|c|c|c|c|}
\hline AD stage & Marker & \multicolumn{6}{|c|}{ Latent dimension } \\
\hline & & First & Second & Third & Fourth & Fifth & Sixth \\
\hline \multirow{6}{*}{$\mathrm{HC}$} & Age & $-0.44(0.003)$ & $0.49(<0.001)$ & $0.01(0.789)$ & $-0.01(0.89)$ & $-0.0(0.983)$ & $0.0(0.941)$ \\
\hline & $\operatorname{CSF} \mathrm{A} \beta$ & $-0.01(0.899)$ & $0.06(0.311)$ & $0.01(0.903)$ & $0.14(0.072)$ & $0.12(0.205)$ & $0.09(0.245)$ \\
\hline & CSF p-tau & $-0.01(0.807)$ & $-0.01(0.808)$ & $-0.01(0.894)$ & $0.06(0.422)$ & $0.06(0.508)$ & $0.1(0.216)$ \\
\hline & CSF t-tau & $-0.0(0.996)$ & $-0.01(0.937)$ & $-0.19(0.076)$ & $0.22(0.016)$ & $0.08(0.485)$ & $0.09(0.418)$ \\
\hline & AD-CSF1 & $-0.13(0.197)$ & $0.02(0.893)$ & $0.02(0.776)$ & $0.04(0.601)$ & $-0.03(0.609)$ & $-0.04(0.638)$ \\
\hline & AD-CSF1 & $0.05(0.497)$ & $-0.0(0.976)$ & $-0.01(0.958)$ & $0.04(0.672)$ & $0.08(0.539)$ & $-0.13(0.224)$ \\
\hline \multirow{6}{*}{ PC } & Age & $-0.42(0.031)$ & $0.28(0.465)$ & $-0.14(0.31)$ & $-0.19(0.211)$ & $-0.1(0.462)$ & $-0.16(0.168)$ \\
\hline & $\operatorname{CSF} \mathrm{A} \beta$ & $-0.01(0.844)$ & $-0.07(0.282)$ & $0.18(0.064)$ & $0.05(0.734)$ & $0.11(0.314)$ & $0.05(0.572)$ \\
\hline & CSF p-tau & $0.05(0.61)$ & $0.03(0.769)$ & $-0.31(0.011)$ & $0.32(0.003)$ & $-0.13(0.349)$ & $-0.04(0.754)$ \\
\hline & CSF t-tau & $0.07(0.452)$ & $-0.0(0.999)$ & $-0.32(0.006)$ & $0.35(<0.001)$ & $-0.2(0.082)$ & $0.02(0.891)$ \\
\hline & AD-CSF1 & $0.02(0.767)$ & $0.08(0.238)$ & $-0.28(0.005)$ & $0.15(0.148)$ & $-0.15(0.202)$ & $-0.07(0.466)$ \\
\hline & AD-CSF1 & $0.05(0.426)$ & $0.01(0.921)$ & $-0.2(0.015)$ & $0.16(0.074)$ & $-0.26(0.002)$ & $-0.01(0.836)$ \\
\hline \multirow{6}{*}{ MCI } & Age & $-0.36(0.072)$ & $0.31(0.196)$ & $-0.12(0.524)$ & $-0.38(<0.001)$ & $-0.08(0.693)$ & $-0.05(0.705)$ \\
\hline & $\operatorname{CSF} \mathrm{A} \beta$ & $0.12(0.205)$ & $-0.09(0.427)$ & $0.26(0.001)$ & $0.01(0.982)$ & $0.06(0.693)$ & $-0.01(0.929)$ \\
\hline & CSF p-tau & $0.04(0.387)$ & $-0.05(0.364)$ & $-0.22(0.006)$ & $0.26(<0.001)$ & $-0.27(<0.001)$ & $-0.19(0.01)$ \\
\hline & CSF t-tau & $0.04(0.468)$ & $-0.03(0.616)$ & $-0.33(<0.001)$ & $0.33(<0.001)$ & $-0.21(0.048)$ & $0.08(0.394)$ \\
\hline & AD-CSF1 & $-0.08(0.436)$ & $-0.04(0.712)$ & $-0.28(<0.001)$ & $0.17(0.068)$ & $-0.18(0.065)$ & $-0.11(0.179)$ \\
\hline & AD-CSF1 & $-0.05(0.412)$ & $0.03(0.623)$ & $-0.33(<0.001)$ & $0.19(0.069)$ & $-0.18(0.114)$ & $0.05(0.601)$ \\
\hline \multirow{6}{*}{$\mathrm{AD}$} & Age & $-0.15(0.25)$ & $0.26(0.092)$ & $0.26(0.181)$ & $-0.48(<0.001)$ & $-0.32(0.122)$ & $0.01(0.96)$ \\
\hline & $\operatorname{CSF} \mathrm{A} \beta$ & $-0.15(0.38)$ & $-0.1(0.576)$ & $0.02(0.771)$ & $0.0(0.977)$ & $-0.09(0.294)$ & $-0.03(0.725)$ \\
\hline & CSF p-tau & $0.12(0.336)$ & $0.05(0.769)$ & $-0.25(0.003)$ & $0.25(0.004)$ & $-0.24(0.003)$ & $-0.12(0.164)$ \\
\hline & CSF t-tau & $0.12(0.229)$ & $-0.09(0.399)$ & $-0.28(0.003)$ & $0.37(<0.001)$ & $-0.15(0.191)$ & $0.17(0.093)$ \\
\hline & AD-CSF1 & $0.18(0.336)$ & $0.12(0.576)$ & $-0.09(0.213)$ & $0.23(<0.001)$ & $-0.26(<0.001)$ & $-0.07(0.386)$ \\
\hline & AD-CSF1 & $0.15(0.238)$ & $-0.06(0.717)$ & $-0.11(0.304)$ & $0.3(0.002)$ & $-0.16(0.104)$ & $0.09(0.384)$ \\
\hline \multirow{6}{*}{$\mathrm{AD}$ continuum } & Age & $-0.31(0.079)$ & $0.29(0.136)$ & $-0.03(0.82)$ & $-0.37(<0.001)$ & $-0.15(0.312)$ & $-0.07(0.544)$ \\
\hline & $\mathrm{CSF} \mathrm{A} \beta$ & $0.15(0.189)$ & $-0.12(0.385)$ & $0.33(<0.001)$ & $0.02(0.958)$ & $0.04(0.893)$ & $0.0(0.976)$ \\
\hline & CSF p-tau & $-0.05(0.43)$ & $0.03(0.723)$ & $\mathbf{- 0 . 3 5}(<0.001)$ & $0.25(0.001)$ & $-0.22(0.058)$ & $-0.14(0.153)$ \\
\hline & CSF t-tau & $-0.09(0.431)$ & $0.01(0.94)$ & $-0.45(<0.001)$ & $0.31(0.005)$ & $-0.18(0.314)$ & $0.08(0.518)$ \\
\hline & AD-CSF1 & $-0.12(0.243)$ & $0.06(0.679)$ & $-0.35(<0.001)$ & $0.17(0.351)$ & $-0.19(0.175)$ & $-0.1(0.335)$ \\
\hline & AD-CSF1 & $-0.15(0.246)$ & $0.06(0.709)$ & $-0.43(<0.001)$ & $0.18(0.517)$ & $-0.18(0.326)$ & $0.03(0.804)$ \\
\hline
\end{tabular}

TABLE V: Volume effect-strength (p-value) on each associated marker of interest separated by AD stages using a single PLS model for AD pathology.

\begin{tabular}{|c|c|c|c|c|c|c|c|}
\hline \multirow[t]{2}{*}{ AD stage } & Marker & \multicolumn{6}{|c|}{ Latent dimension } \\
\hline & & First & Second & Third & Fourth & Fifth & Sixth \\
\hline \multirow{6}{*}{$\mathrm{HC}$} & Age & $-0.42(0.026)$ & $0.45(0.011)$ & $-0.03(0.85)$ & $-0.18(0.033)$ & $-0.03(0.752)$ & $-0.1(0.216)$ \\
\hline & $\operatorname{CSF} \mathrm{A} \beta$ & $0.03(0.739)$ & $0.07(0.335)$ & $0.04(0.611)$ & $0.1(0.24)$ & $0.02(0.849)$ & $-0.13(0.06)$ \\
\hline & CSF p-tau & $-0.01(0.864)$ & $0.01(0.865)$ & $0.09(0.308)$ & $0.02(0.795)$ & $-0.05(0.512)$ & $0.11(0.252)$ \\
\hline & CSF t-tau & $0.03(0.892)$ & $0.19(0.064)$ & $-0.08(0.292)$ & $0.1(0.179)$ & $-0.01(0.941)$ & $0.08(0.414)$ \\
\hline & AD-CSF1 & $-0.13(0.16)$ & $0.15(0.114)$ & $0.22(0.005)$ & $-0.07(0.493)$ & $0.01(0.927)$ & $0.09(0.217)$ \\
\hline & AD-CSF1 & $0.11(0.267)$ & $-0.0(0.98)$ & $-0.03(0.754)$ & $0.18(0.015)$ & $0.16(0.03)$ & $0.06(0.561)$ \\
\hline \multirow{6}{*}{$\mathrm{PC}$} & Age & $-0.4(0.055)$ & $0.15(0.676)$ & $-0.33(0.147)$ & $-0.2(0.588)$ & $-0.07(0.668)$ & $-0.05(0.701)$ \\
\hline & $\operatorname{CSF} \mathrm{A} \beta$ & $0.02(0.672)$ & $-0.14(0.087)$ & $0.14(0.157)$ & $-0.06(0.492)$ & $0.13(0.318)$ & $-0.01(0.937)$ \\
\hline & CSF p-tau & $-0.13(0.2)$ & $0.18(0.166)$ & $-0.35(0.011)$ & $0.34(0.003)$ & $-0.06(0.8)$ & $-0.02(0.923)$ \\
\hline & CSF t-tau & $-0.11(0.254)$ & $0.18(0.162)$ & $-0.35(0.011)$ & $0.35(<0.001)$ & $0.07(0.732)$ & $0.17(0.268)$ \\
\hline & AD-CSF1 & $-0.14(0.174)$ & $0.14(0.304)$ & $-0.34(0.008)$ & $0.17(0.245)$ & $-0.13(0.55)$ & $0.05(0.742)$ \\
\hline & AD-CSF1 & $-0.04(0.46)$ & $0.14(0.151)$ & $-0.24(0.025)$ & $0.17(0.095)$ & $-0.01(0.977)$ & $0.22(0.059)$ \\
\hline \multirow{6}{*}{ MCI } & Age & $-0.41(0.026)$ & $0.13(0.723)$ & $-0.19(0.551)$ & $-0.36(0.03)$ & $0.04(0.815)$ & $-0.08(0.394)$ \\
\hline & $\operatorname{CSF} \mathrm{A} \beta$ & $0.15(0.063)$ & $-0.06(0.584)$ & $0.12(0.095)$ & $-0.0(0.988)$ & $0.02(0.749)$ & $0.24(<0.001)$ \\
\hline & CSF p-tau & $-0.02(0.551)$ & $-0.01(0.894)$ & $-0.21(0.006)$ & $0.27(<0.001)$ & $0.22(0.009)$ & $-0.08(0.422)$ \\
\hline & CSF t-tau & $-0.11(0.184)$ & $0.08(0.401)$ & $-0.26(0.001)$ & $0.3(<0.001)$ & $0.3(<0.001)$ & $-0.07(0.455)$ \\
\hline & AD-CSF1 & $-0.14(0.129)$ & $0.02(0.858)$ & $-0.23(<0.001)$ & $0.15(0.065)$ & $0.09(0.435)$ & $-0.11(0.152)$ \\
\hline & AD-CSF1 & $-0.17(0.102)$ & $0.1(0.433)$ & $-0.25(0.002)$ & $0.21(0.009)$ & $0.14(0.193)$ & $-0.1(0.244)$ \\
\hline \multirow{6}{*}{$\mathrm{AD}$} & Age & $-0.06(0.5)$ & $0.17(0.318)$ & $0.19(0.556)$ & $-0.6(<0.001)$ & $0.21(0.568)$ & $0.01(0.942)$ \\
\hline & $\operatorname{CSF} \mathrm{A} \beta$ & $-0.12(0.49)$ & $-0.08(0.482)$ & $-0.06(0.508)$ & $-0.06(0.508)$ & $0.03(0.733)$ & $0.19(0.01)$ \\
\hline & CSF p-tau & $-0.06(0.541)$ & $-0.05(0.555)$ & $-0.25(0.004)$ & $0.21(0.022)$ & $0.2(0.066)$ & $-0.2(0.017)$ \\
\hline & CSF t-tau & $-0.15(0.533)$ & $-0.13(0.31)$ & $-0.24(0.003)$ & $0.27(0.002)$ & $0.19(0.112)$ & $0.06(0.569)$ \\
\hline & AD-CSF1 & $0.0(0.911)$ & $-0.08(0.182)$ & $-0.11(0.075)$ & $0.15(0.017)$ & $0.12(0.133)$ & $-0.18(0.016)$ \\
\hline & AD-CSF1 & $0.03(0.422)$ & $-0.09(0.181)$ & $-0.07(0.551)$ & $0.25(<0.001)$ & $0.13(0.255)$ & $-0.02(0.88)$ \\
\hline \multirow{6}{*}{$\mathrm{AD}$ continuum } & Age & $-0.31(0.053)$ & $0.15(0.609)$ & $-0.11(0.723)$ & $\begin{array}{c}-0.4(<0.001) \\
\end{array}$ & $0.07(0.733)$ & $-0.04(0.667)$ \\
\hline & $\mathrm{CSF} \mathrm{A} \beta$ & $0.18(0.068)$ & $-0.08(0.644)$ & $0.25(0.003)$ & $-0.03(0.924)$ & $0.02(0.883)$ & $0.21(<0.001)$ \\
\hline & CSF p-tau & $-0.17(0.187)$ & $0.02(0.912)$ & $\mathbf{- 0 . 3 5}(<0.001)$ & $0.25(0.016)$ & $0.17(0.316)$ & $-0.14(0.174)$ \\
\hline & CSF t-tau & $-0.25(0.136)$ & $0.03(0.912)$ & $-0.42(<0.001)$ & $0.28(0.046)$ & $0.23(0.161)$ & $-0.05(0.692)$ \\
\hline & AD-CSF1 & $-0.22(0.117)$ & $0.03(0.904)$ & $-0.35(<0.001)$ & $0.15(0.511)$ & $0.07(0.716)$ & $-0.13(0.145)$ \\
\hline & AD-CSF1 & $-0.24(0.099)$ & $0.07(0.794)$ & $-0.39(<0.001)$ & $0.19(0.356)$ & $0.13(0.507)$ & $-0.07(0.499)$ \\
\hline
\end{tabular}

TABLE VI: Cortical thickness effect-strength (p-value) on each associated marker of interest separated by AD stages using a single PLS model for AD pathology. 


\begin{tabular}{|c|c|c|c|c|c|c|c|}
\hline AD stage & Marker & \multicolumn{6}{|c|}{ Latent dimension } \\
\hline & & First & Second & Third & Fourth & Fifth & Sixth \\
\hline \multirow{6}{*}{$\mathrm{HC}$} & Age & $-0.44(0.01)$ & $0.49(<0.001)$ & $-0.07(0.22)$ & $-0.01(0.88)$ & $-0.14(0.01)$ & $0.1(0.06)$ \\
\hline & $\operatorname{CSF} \mathrm{A} \beta$ & $-0.01(0.9)$ & $0.06(0.29)$ & $-0.32(<0.001)$ & $0.36(<0.001)$ & $-0.23(<0.001)$ & $-0.05(0.59)$ \\
\hline & CSF p-tau & $-0.01(0.81)$ & $-0.01(0.8)$ & $-0.27(<0.001)$ & $-0.22(<0.001)$ & $-0.24(<0.001)$ & $-0.25(<0.001)$ \\
\hline & CSF t-tau & $-0.0(0.99)$ & $-0.01(0.93)$ & $-0.52(<0.001)$ & $-0.2(0.07)$ & $-0.28(<0.001)$ & $0.28(<0.001)$ \\
\hline & AD-CSF1 & $-0.13(0.2)$ & $0.02(0.88)$ & $0.01(0.87)$ & $-0.08(0.29)$ & $-0.09(0.19)$ & $-0.16(0.02)$ \\
\hline & AD-CSF1 & $0.05(0.5)$ & $-0.0(0.98)$ & $-0.16(0.13)$ & $-0.23(0.02)$ & $0.07(0.55)$ & $0.14(0.21)$ \\
\hline \multirow{6}{*}{ PC } & Age & $-0.42(0.04)$ & $0.28(0.45)$ & $0.1(0.5)$ & $0.12(0.44)$ & $-0.15(0.28)$ & $0.25(0.04)$ \\
\hline & $\operatorname{CSF} \mathrm{A} \beta$ & $-0.01(0.85)$ & $-0.07(0.28)$ & $-0.18(0.04)$ & $-0.06(0.61)$ & $0.33(<0.001)$ & $-0.19(0.03)$ \\
\hline & CSF p-tau & $0.05(0.61)$ & $0.03(0.8)$ & $0.6(<0.001)$ & $0.35(<0.001)$ & $-0.22(0.08)$ & $-0.22(0.09)$ \\
\hline & CSF t-tau & $0.07(0.48)$ & $-0.0(1.0)$ & $0.58(<0.001)$ & $0.43(<0.001)$ & $-0.21(0.1)$ & $-0.18(0.14)$ \\
\hline & AD-CSF1 & $0.02(0.79)$ & $0.08(0.26)$ & $0.39(<0.001)$ & $0.28(<0.001)$ & $-0.29(<0.001)$ & $0.03(0.8)$ \\
\hline & AD-CSF1 & $0.05(0.47)$ & $0.01(0.92)$ & $0.33(<0.001)$ & $0.25(0.01)$ & $-0.27(<0.001)$ & $0.02(0.82)$ \\
\hline \multirow{6}{*}{ MCI } & Age & $-0.36(0.07)$ & $0.31(0.18)$ & $0.02(0.94)$ & $0.37(<0.001)$ & $0.08(0.61)$ & $0.1(0.52)$ \\
\hline & $\operatorname{CSF} \mathrm{A} \beta$ & $0.12(0.21)$ & $-0.09(0.4)$ & $0.25(0.01)$ & $-0.09(0.6)$ & $-0.15(0.14)$ & $0.01(0.94)$ \\
\hline & CSF p-tau & $0.04(0.39)$ & $-0.05(0.36)$ & $-0.37(<0.001)$ & $-0.27(<0.001)$ & $0.11(0.16)$ & $0.27(<0.001)$ \\
\hline & CSF t-tau & $0.04(0.5)$ & $-0.03(0.64)$ & $-0.47(<0.001)$ & $-0.28(<0.001)$ & $0.24(<0.001)$ & $0.03(0.75)$ \\
\hline & AD-CSF1 & $-0.08(0.43)$ & $-0.04(0.73)$ & $-0.36(<0.001)$ & $-0.11(0.31)$ & $0.11(0.23)$ & $0.15(0.08)$ \\
\hline & AD-CSF1 & $-0.05(0.44)$ & $0.03(0.61)$ & $-0.4(<0.001)$ & $-0.12(0.27)$ & $0.21(0.01)$ & $0.07(0.48)$ \\
\hline \multirow{6}{*}{$\mathrm{AD}$} & Age & $-0.15(0.27)$ & $0.26(0.08)$ & $0.48(<0.001)$ & $-0.07(0.73)$ & $-0.22(0.27)$ & $-0.21(0.28)$ \\
\hline & $\mathrm{CSF} \mathrm{A} \beta$ & $-0.15(0.36)$ & $-0.1(0.62)$ & $-0.02(0.81)$ & $-0.04(0.59)$ & $-0.11(0.23)$ & $0.02(0.85)$ \\
\hline & CSF p-tau & $0.12(0.32)$ & $0.05(0.79)$ & $-0.41(<0.001)$ & $-0.31(<0.001)$ & $-0.32(<0.001)$ & $0.07(0.4)$ \\
\hline & CSF t-tau & $0.12(0.23)$ & $-0.09(0.42)$ & $-0.49(<0.001)$ & $-0.32(<0.001)$ & $0.09(0.4)$ & $-0.27(<0.001)$ \\
\hline & AD-CSF1 & $0.18(0.34)$ & $0.12(0.58)$ & $-0.25(<0.001)$ & $-0.25(<0.001)$ & $-0.17(0.02)$ & $0.06(0.41)$ \\
\hline & AD-CSF1 & $0.15(0.25)$ & $-0.06(0.73)$ & $-0.33(<0.001)$ & $-0.24(0.02)$ & $0.09(0.34)$ & $-0.2(0.05)$ \\
\hline
\end{tabular}

TABLE VII: Volume effect-strength (p-value) on each associated marker of interest separated by AD stages using multiple PLS models for $\mathrm{AD}$ pathology.

\begin{tabular}{|c|c|c|c|c|c|c|c|}
\hline AD stage & Marker & \multicolumn{6}{|c|}{ Latent dimension } \\
\hline & & First & Second & Third & Fourth & Fifth & Sixth \\
\hline \multirow{6}{*}{$\mathrm{HC}$} & Age & $-0.42(0.02)$ & $0.45(0.01)$ & $-0.05(0.64)$ & $-0.09(0.43)$ & $-0.13(0.14)$ & $-0.16(0.06)$ \\
\hline & $\operatorname{CSF} \mathrm{A} \beta$ & $0.03(0.74)$ & $0.07(0.33)$ & $0.17(0.01)$ & $-0.37(<0.001)$ & $0.31(<0.001)$ & $-0.09(0.23)$ \\
\hline & CSF p-tau & $-0.01(0.83)$ & $0.01(0.84)$ & $0.42(<0.001)$ & $-0.1(0.33)$ & $-0.18(0.04)$ & $-0.22(0.01)$ \\
\hline & CSF t-tau & $0.03(0.88)$ & $0.19(0.06)$ & $0.44(<0.001)$ & $-0.28(<0.001)$ & $-0.19(0.02)$ & $0.2(0.02)$ \\
\hline & AD-CSF1 & $-0.13(0.14)$ & $0.15(0.12)$ & $0.11(0.22)$ & $0.13(0.14)$ & $-0.06(0.45)$ & $-0.12(0.12)$ \\
\hline & AD-CSF1 & $0.11(0.28)$ & $-0.0(0.98)$ & $0.16(0.05)$ & $-0.05(0.63)$ & $0.02(0.8)$ & $0.14(0.09)$ \\
\hline \multirow{6}{*}{$\mathrm{PC}$} & Age & $-0.4(0.06)$ & $0.15(0.65)$ & $0.22(0.39)$ & $0.15(0.81)$ & $-0.24(0.26)$ & $-0.07(0.66)$ \\
\hline & $\operatorname{CSF} \mathrm{A} \beta$ & $0.02(0.67)$ & $-0.14(0.08)$ & $-0.24(0.02)$ & $-0.01(0.89)$ & $-0.0(0.98)$ & $-0.33(<0.001)$ \\
\hline & CSF p-tau & $-0.13(0.17)$ & $0.18(0.16)$ & $0.56(<0.001)$ & $-0.26(0.03)$ & $-0.23(0.2)$ & $0.2(0.23)$ \\
\hline & CSF t-tau & $-0.11(0.23)$ & $0.18(0.17)$ & $0.52(<0.001)$ & $-0.25(0.03)$ & $-0.25(0.11)$ & $-0.05(0.76)$ \\
\hline & AD-CSF1 & $-0.14(0.19)$ & $0.14(0.31)$ & $0.49(<0.001)$ & $-0.05(0.87)$ & $-0.14(0.51)$ & $0.27(0.05)$ \\
\hline & AD-CSF1 & $-0.04(0.44)$ & $0.14(0.16)$ & $0.35(<0.001)$ & $-0.1(0.37)$ & $-0.16(0.23)$ & $0.12(0.39)$ \\
\hline \multirow{6}{*}{ MCI } & Age & $-0.41(0.03)$ & $0.13(0.7)$ & $0.04(0.91)$ & $-0.3(0.28)$ & $-0.3(0.07)$ & $0.01(0.88)$ \\
\hline & $\operatorname{CSF} \mathrm{A} \beta$ & $0.15(0.07)$ & $-0.06(0.57)$ & $0.08(0.31)$ & $0.05(0.54)$ & $0.17(<0.001)$ & $-0.36(<0.001)$ \\
\hline & CSF p-tau & $-0.02(0.56)$ & $-0.01(0.91)$ & $-0.42(<0.001)$ & $0.2(<0.001)$ & $-0.18(0.02)$ & $0.05(0.57)$ \\
\hline & CSF t-tau & $-0.11(0.19)$ & $0.08(0.41)$ & $-0.45(<0.001)$ & $0.19(<0.001)$ & $-0.22(0.01)$ & $0.12(0.2)$ \\
\hline & AD-CSF1 & $-0.14(0.14)$ & $0.02(0.86)$ & $-0.3(<0.001)$ & $0.03(0.77)$ & $-0.16(0.05)$ & $0.21(<0.001)$ \\
\hline & AD-CSF1 & $-0.17(0.1)$ & $0.1(0.45)$ & $-0.34(<0.001)$ & $0.06(0.4)$ & $-0.18(0.05)$ & $0.24(<0.001)$ \\
\hline \multirow{6}{*}{$\mathrm{AD}$} & Age & $-0.06(0.49)$ & $0.17(0.32)$ & $0.49(0.02)$ & $-0.07(0.94)$ & $0.08(0.78)$ & $-0.33(0.06)$ \\
\hline & $\mathrm{CSF} \mathrm{A} \beta$ & $-0.12(0.5)$ & $-0.08(0.5)$ & $-0.06(0.53)$ & $-0.09(0.34)$ & $0.05(0.55)$ & $-0.03(0.66)$ \\
\hline & CSF p-tau & $-0.06(0.58)$ & $-0.05(0.52)$ & $-0.39(<0.001)$ & $-0.3(<0.001)$ & $-0.25(0.01)$ & $-0.17(0.06)$ \\
\hline & CSF t-tau & $-0.15(0.57)$ & $-0.13(0.31)$ & $-0.4(<0.001)$ & $-0.35(<0.001)$ & $-0.03(0.78)$ & $0.18(0.08)$ \\
\hline & AD-CSF1 & $0.0(0.91)$ & $-0.08(0.2)$ & $-0.24(<0.001)$ & $-0.17(0.01)$ & $-0.26(<0.001)$ & $-0.13(0.08)$ \\
\hline & AD-CSF1 & $0.03(0.4)$ & $-0.09(0.17)$ & $-0.25(<0.001)$ & $-0.33(<0.001)$ & $-0.08(0.4)$ & $0.2(0.01)$ \\
\hline
\end{tabular}

TABLE VIII: Cortical thickness effect-strength (p-value) on each associated marker of interest separated by AD stages using multiple PLS models for AD pathology. 


\section{REFERENCES}

[1] Peters, R. "Aging and the brain." Postgraduate Med. Journal 82.964 (2006): 84-88.

[2] Aisen, Paul S., et al. "On the path to 2025: understanding the Alzheimer's disease continuum." Alzheimer's research \& therapy 9.1 (2017): 60.

[3] Molinuevo, Jose L., et al. "The AD-CSF-index discriminates Alzheimer's disease patients from healthy controls: a validation study." Journal of Alzheimer's Disease 36.1 (2013): 67-77.

[4] Dubois, Bruno, et al. "Preclinical Alzheimer's disease: definition, natural history, and diagnostic criteria." Alzheimer's \& Dementia 12.3 (2016): 292-323.

[5] Jack Jr, Clifford R., et al. "NIA-AA Research Framework: Toward a biological definition of Alzheimer's disease." Alzheimer's \& Dementia 14.4 (2018): 535-562.

[6] Ossenkoppele, Rik, et al. "Discriminative accuracy of [18F] flortaucipir positron emission tomography for Alzheimer disease vs other neurodegenerative disorders." Jama 320.11 (2018): 1151-1162.

[7] Fjell, Anders M., et al. "What is normal in normal aging? Effects of aging, amyloid and Alzheimer's disease on the cerebral cortex and the hippocampus." Progress in neurobiology 117 (2014): 20-40.

[8] Wirth, Miranka, et al. "Alzheimer's disease neurodegenerative biomarkers are associated with decreased cognitive function but not $\beta$-amyloid in cognitively normal older individuals.” Journal of Neuroscience 33.13 (2013): 5553-5563

[9] Mungas, D., et al. "Volumetric MRI predicts rate of cognitive decline related to AD and cerebrovascular disease." Neurology 59.6 (2002): 867873.

[10] Casamitjana, Adrià, et al. "MRI-based screening of preclinical Alzheimers disease for prevention clinical trials." Journal of Alzheimer's Disease Preprint (2018): 1-14.

[11] Ossenkoppele, Rik, et al. "Associations between tau, A $\beta$, and cortical thickness with cognition in Alzheimer disease." Neurology 92.6 (2019): e601-e612.

[12] Iaccarino, Leonardo, et al. "Local and distant relationships between amyloid, tau and neurodegeneration in Alzheimer's Disease." NeuroImage: Clinical 17 (2018): 452-464.

[13] Kanai, Ryota, and Geraint Rees. "The structural basis of inter-individual differences in human behaviour and cognition." Nature Reviews Neuroscience 12.4 (2011): 231

[14] Krishnan, Anjali, et al. "Partial Least Squares (PLS) methods for neuroimaging: a tutorial and review." Neuroimage 56.2 (2011): 455-475.

[15] Lorenzi, Marco, et al. "Partial least squares modelling for imaginggenetics in Alzheimer's disease: Plausibility and generalization." Biomedical Imaging (ISBI), 2016 IEEE 13th International Symposium on. IEEE, 2016.

[16] Konukoglu, Ender, et al. "Multivariate statistical analysis of diffusion imaging parameters using partial least squares: Application to white matter variations in Alzheimer's disease.” Neuroimage 134 (2016): 573 586.

[17] Westman, Eric, et al. "AddNeuroMed and ADNI: similar patterns of Alzheimer's atrophy and automated MRI classification accuracy in Europe and North America." Neuroimage 58.3 (2011): 818-828.

[18] Wachinger, Christian, Anna Rieckmann, and Martin Reuter. "Latent Processes Governing Neuroanatomical Change in Aging and Dementia." MICCAI. Springer, Cham, 2017.

[19] Rathore, Saima, et al. "A review on neuroimaging-based classification studies and associated feature extraction methods for Alzheimer's disease and its prodromal stages." NeuroImage 155 (2017): 530-548.

[20] Jack, Clifford R., et al. "A/T/N: an unbiased descriptive classification scheme for Alzheimer disease biomarkers." Neurology 87.5 (2016): 539547.

[21] ten Kate, Mara, et al. MRI predictors of amyloid pathology: results from the EMIF-AD Multimodal Biomarker Discovery study. Alzheimer's research \& therapy 10.1 (2018): 100.

[22] Vemuri, P., et al. "MRI and CSF biomarkers in normal, MCI, and AD subjects: diagnostic discrimination and cognitive correlations." Neurology 73.4 (2009): 287-293.

[23] Vemuri, P., et al. "MRI and CSF biomarkers in normal, MCI, and AD subjects: predicting future clinical change.” Neurology 73.4 (2009): 294 301.

[24] Fjell, Anders M., et al. "CSF biomarkers in prediction of cerebral and clinical change in mild cognitive impairment and Alzheimer's disease." Journal of Neuroscience 30.6 (2010): 2088-2101.
[25] Abdi, Herv. "Partial least squares regression and projection on latent structure regression (PLS Regression)." Wiley Interdisciplinary Reviews: Computational Statistics 2.1 (2010): 97-106.

[26] Good, Phillip I. "Permutation, parametric, and bootstrap tests of hypotheses (Springer series in statistics)." (2004).

[27] Jack, Clifford R., et al. "NIA-AA Research Framework: Toward a biological definition of Alzheimer's disease." Alzheimer's \& Dementia 14.4 (2018): 535-562.

[28] Jack Jr, Clifford R., et al. "Introduction to the recommendations from the National Institute on Aging-Alzheimer's Association workgroups on diagnostic guidelines for Alzheimer's disease.” Alzheimer's \& Dementia 7.3 (2011): 257-262.

[29] Shaw, Leslie M., et al. "Cerebrospinal fluid biomarker signature in Alzheimer's disease neuroimaging initiative subjects." Annals of neurology 65.4 (2009): 403-413.

[30] Fischl, Bruce, et al. "Whole brain segmentation: automated labeling of neuroanatomical structures in the human brain." Neuron 33.3 (2002): 341-355.

[31] Fischl, Bruce, et al. "Automatically parcellating the human cerebral cortex." Cerebral cortex 14.1 (2004): 11-22.

[32] Varoquaux, Gaël, et al. "Assessing and tuning brain decoders: crossvalidation, caveats, and guidelines." NeuroImage 145 (2017): 166-179.

[33] Albert, Marilyn S., et al. "The diagnosis of mild cognitive impairment due to Alzheimer's disease: Recommendations from the National Institute on Aging-Alzheimer's Association workgroups on diagnostic guidelines for Alzheimer's disease.” Alzheimer's \& dementia 7.3 (2011): 270-279.

[34] Falcon, Carles, et al. "Longitudinal structural cerebral changes related to core CSF biomarkers in preclinical Alzheimer's disease: A study of two independent datasets." NeuroImage: Clinical 19 (2018): 190-201.

[35] FennemaNotestine, Christine, et al. "Structural MRI biomarkers for preclinical and mild Alzheimer's disease." Human brain mapping 30.10 (2009): 3238-3253.

[36] Bondi, Mark W., et al. "fMRI evidence of compensatory mechanisms in older adults at genetic risk for Alzheimer disease." Neurology 64.3 (2005): 501-508.

[37] Qi, Zhigang, et al. "Impairment and compensation coexist in amnestic MCI default mode network." Neuroimage 50.1 (2010): 48-55.

[38] Salat, David H., et al. "Thinning of the cerebral cortex in aging." Cerebral cortex 14.7 (2004): 721-730.

[39] Hutton, Chloe, et al. "A comparison between voxel-based cortical thickness and voxel-based morphometry in normal aging." Neuroimage 48.2 (2009): 371-380.

[40] Thambisetty, Madhav, et al. "Longitudinal changes in cortical thickness associated with normal aging." Neuroimage 52.4 (2010): 1215-1223.

[41] Tosun, Duygu, et al. "Relations between brain tissue loss, CSF biomarkers, and the ApoE genetic profile: a longitudinal MRI study." Neurobiology of aging 31.8 (2010): 1340-1354.

[42] Moradi, Elaheh, et al. "Machine learning framework for early MRIbased Alzheimer's conversion prediction in MCI subjects." Neuroimage 104 (2015): 398-412.

[43] Young, Alexandra L., et al. "Uncovering the heterogeneity and temporal complexity of neurodegenerative diseases with Subtype and Stage Inference.” Nature communications 9.1 (2018): 4273 